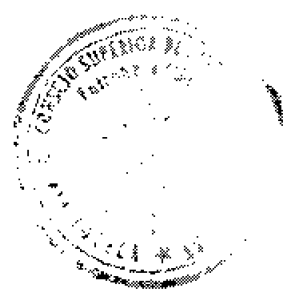

$634-38$

\title{
estudio de los factores térmicos del horno en zigzag
}

Una serie de cualidades favorables dentro de los medios técnicos y económicos que poseemos en España han originađo la dífusión de los hornos de cámaras en zigzag, en la industria de la cerámica de la construcción.

Su concepción, en efecto, es tal, que hace de él un horno de entretenimiento fácil, de conđucción cómoda; y dentro de estas propiedades, de un rendimiento térmico aceptable, al reducir al mínimo las pérdidas con la disposición de sus cámaras, las cuales presentan al exterior una superficie relativamente reducida en comparación con la superficie total de las cámaras, la cual está sometida a las distintas temperaturas a las que dentro de una ley determinada se calienta el material cerámico que en ellas se cuece.

Las pérdidas por aberturas o intersticios son prácticamente despreciables, debido a que las elevadas temperaturas de la zona de fuego quedan a una distancia considerable de las partes del horno en comunicación dirrecta con el exterior (entrada y salida de aire, humos y elementos cocidos) y a que las bocas de alimentación son de una superficie reducida y se puede asegurar en ellas un cierre cómodo y eflcaz.

Por la característica del horno, Ia cale-
A. ALAMAN

ingeniero industrial facción del aire comburente-no sólo posible, sino necesaria, al atravesar los apilados cocidos y un período de enfriamiento-se efectúa con facilidad y eficacia, alcanzando este aire temperaturas elevadas que favorecen enormemente la combustiốn del carbón alimentado por las bocas de la zona de fuego, lo que permite utilizar carbones de calidades inferiores.

Las calorias almacenadas en los elementos cocidos son excesivas para el calentamiento del aire de la combustión, por lo que se impone un sistema de recuperación de las mismas, que permite utilizarlas en otra instalación de la fábrica en donde sean necesarias unas calorías suministradas por un agente que no se encuentre a temperatura elevada. Esta recuperación se efectúa por el aire, que a una temperatura variable se destina normalmente al secadero de los productos moldeados. Esto, al permitir a la vez la evacuación de los productos cocidos, a una temperatura muy próxima al ambiente, supone un término positivo que tiene un valor considerable a la hora de efectuar el balance térmico $\mathrm{y}$ el rendimiento de la instalación, reduciendo, al mínimo, las calorías perdidas por los elementos que, calientes, se enfrian en el exterior.

La circunstancia de que los productos secos deban calentarse paulatinamente, 
favorece la recuperación del calor sensible de los productos de la combustión, que ceden sus calorías a los productos que se van a cocer, evacuándose aquéllos al exterior a temperaturas considerablemente bajas.

Aparte de las consideraciones térmicas, estos hornos tienen unas ventajas que se traducen en un ahorro de dinero, tanto en la instalación de los mismos como en su entretenimiento.

Su construcción es sencilla y económica. Esta sencillez se debe en gran parte a los materiales que intervienen en él, ladrillos y un aislante tan barato y de cómodo manejo, por ejemplo, como lo es la arena. Las cargas y temperaturas que soportan los ladrillos son relativamente bajas, por lo que no es preciso utilizar ladrillos refractarios de calidad.

El entretenimiento de este horno es económico. Las partes móviles del mismo son reducidas, de manejo fácil y todas ellas alejadas de las zonas de temperaturas elevadas. Los elementos más delicados los constituyen las válvulas y los ventiladores extractores; $y$ unas y otros, con una revisión periódica, pueden mantenerse en perfecto estado por tiempo indefinido, ya que sus condiciones de trabajo no son duras en ningún sentido.

La obra del horno, cuyas partes más delicadas están a la vista, no requiere en general una revisión especial, sino que, procurando corregir los desperfectos que en el revestimiento o en la obra de Iadrillo pudiesen apreciarse durante los períodos en los que cada cámara puede ser visitada, se evitarán fácilmente reparaciones de envergadura considerable.

Frente a estas indudables ventajas aparecen una serie de inconvenientes, cuya mayor o menor importancia dependerá muchas veces de las condiciones particulares de instalación del horno.

La carga y descarga del horno ha de hacerse manualmente, por lo menos en parte, lo que requiere mano de obra, tiempo y cuidado que la automatización elimina en gran porcentaje.

La carga del horno debe ser distribuída cuidadosamente. En efecto, por la forma de éste ha de procederse al encañado, procurando distribuir los productos de manera que ofrezcan mayor dificultad al paso de los gases en los lugares donde la corriente de aire o gases tenga tendencia a circular con mayor facilidad. Lo ideal sería una velocidad de circulación uniforme en cada sección y en cada momento, pero las condiciones teóricas son prácticamente imposibles de ser alcanzadas, con lo que no se pueden evitar zonas sobrecalentadas y zonas frías, con la consiguiente falta de uniformidad en la calidad del producto cocido. Estas diferencias se atenúan disponiendo entradas auxiliares de aire, o conductos auxiliares por los que los gases, con menos pérdida de carga, se conducen a los lugares más in-

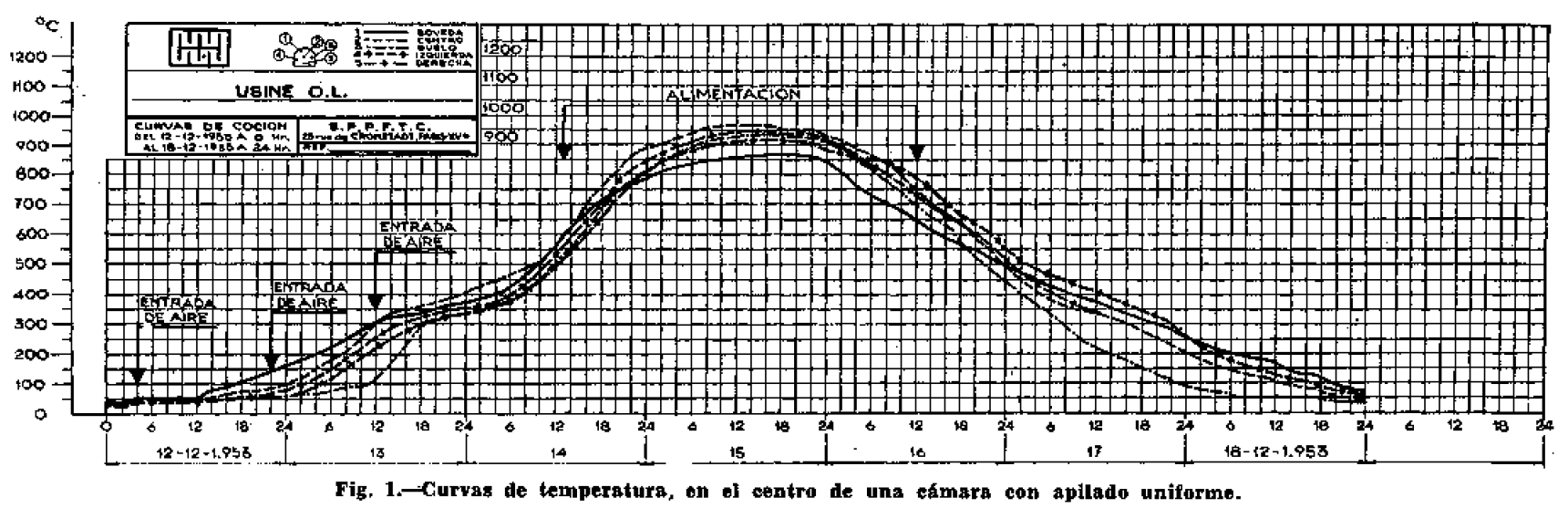

Fig. 1. Curvas de temperatura, on ol centro de una camara con apilado uniforme. 
teresantes. Como consecuencia de esto no es difícil encontrar diferencias de alrededor de $100^{\circ} \mathrm{C}$ en el centro de una cámara en donde lógicamente la distribución y uniformidad es mayor, y diferencias del orden de $250^{\circ} \mathrm{C}$ en los extremos de las cámaras.

A título informativo se dan las curvas de cocción de un horno en rigzag, con las temperaturas tomadas en diversos puntos de la sección en el centro de una cámara.

Por otra parte, si bien las condiciones de combustión del carbón alimentado por las bocas se ve favorecida intensamente por las altas temperaturas del aire comcuidado-ni mucho menos-, ya que es el cuidado ni mucho menos, ya que es el propio apilado de ladrillo dispuesto con vistas a la transmisión eficiente y uniforme del calor, y no para que la combustión se realice en las mejores condiciones. Consecuencia importante de lo anterior es el porcentaje de inquemados, cifra de difícil evaluación, especialmente a priori, que depende de muchos factores particulares -horno, hornero, tipo de combustible y tipo de producto-y que, desde luego, hay que tener presente en un balance térmico.

El aislamiento térmico de las cámaras con el exterior en estos hornos puede hacerse tan perfecto como se quiera, y con materiales económicos. Para lograr un ahorro de las calorías perdidas sin encarecerlos considerablemente se recurre a espesores apreciables de pared o techo, introduciendo, en los huecos dejados al efecto, arena como aislante térmico, aislante que empleado en espesores convenientes resulta económico y eficaz. Si bien, como se dice, las pérdidas por radiación pueden reducirse notablemente, ya que la temperatura exterior de un horno calculado con un aislamiento adecuado es prácticamente la del ambiente, estas grandes masas aislantes almacenan unas calorías, que si en parte son cedidas en el período de enfriamiento, muchas de ellas se pierden al vaciar las cámaras, que antes de volverse a llenar quedan prácticamente a la temperatura ambiente.
En la balanza de vicios y virtudes del horno en zigzag, éstas pesan más que aquéllas, tanto bajo el punto de vista técnico como en el aspecto económico, y esta inclinación del flel ha sido la causante de su aceptación en la industria.

\section{CALCULOS TERMICOS EN EL PROYECTO}

Vamos a considerar el caso más general, es decir, un horno con extracción de aire caliente para aprovechamiento posterior. Para mayor sencillez supondremos el horno con un solo fuego. Si fuera de varios, el problema, con las ligeras variaciones procedentes de las pérdidas por transmisión, dependientes en cada horno de la distribución de temperaturas en él, se reduciría a multiplicar por $n$ los resultados obtenidos en el cálculo. Este cálculo no pretende ser riguroso, sino aproximado: con un grado de aproximación suficiente para proporcionar datos dignos de ser considerados como reales sin cometer errores groseros. Nos apoyaremos en algunos apartados en datos confirmados por la experiencia, que merecen crédito para este tipo de hornos y que simplifican notablemente las operaciones.

No se tiene en cuenta el período transitorio de puesta en funcionamiento o de encendido después de ser apagado por cualquier circunstancia. En este horno, que funciona continuamente, por su sencillez, solidez $y$ facilidad de manejo raramente se da una interrupción de marcha, por lo que estos períodos excepcionales se diluyen frente al tiempo de funcionamiento continuo.

Es cómodo referir los cálculos siguientes al intervalo de tiempo en el que, como término medio, se produce una cámara, ya que es el período de tiempo en el cual se repiten en el horno todos los fenómenos $\mathrm{y}$ distribución de temperaturas, aunque con una cámara de adelanto, naturalmente.

El intercambio de calor que en un hor- 
teresantes. Como consecuencia de esto no es difícil encontrar diferencias de alrededor de $100^{\circ} \mathrm{C}$ en el centro de una cámara en donde lógicamente la distribución y uniformidad es mayor, y diferencias del orden de $250^{\circ} \mathrm{C}$ en los extremos de las cámaras.

A título informativo se dan las curvas de cocción de un horno en rigzag, con las temperaturas tomadas en diversos puntos de la sección en el centro de una cámara.

Por otra parte, si bien las condiciones de combustión del carbón alimentado por las bocas se ve favorecida intensamente por las altas temperaturas del aire comcuidado-ni mucho menos-, ya que es el cuidado ni mucho menos, ya que es el propio apilado de ladrillo dispuesto con vistas a la transmisión eficiente y uniforme del calor, y no para que la combustión se realice en las mejores condiciones. Consecuencia importante de lo anterior es el porcentaje de inquemados, cifra de difícil evaluación, especialmente a priori, que depende de muchos factores particulares -horno, hornero, tipo de combustible y tipo de producto-y que, desde luego, hay que tener presente en un balance térmico.

El aislamiento térmico de las cámaras con el exterior en estos hornos puede hacerse tan perfecto como se quiera, y con materiales económicos. Para lograr un ahorro de las calorías perdidas sin encarecerlos considerablemente se recurre a espesores apreciables de pared o techo, introduciendo, en los huecos dejados al efecto, arena como aislante térmico, aislante que empleado en espesores convenientes resulta económico y eficaz. Si bien, como se dice, las pérdidas por radiación pueden reducirse notablemente, ya que la temperatura exterior de un horno calculado con un aislamiento adecuado es prácticamente la del ambiente, estas grandes masas aislantes almacenan unas calorías, que si en parte son cedidas en el período de enfriamiento, muchas de ellas se pierden al vaciar las cámaras, que antes de volverse a llenar quedan prácticamente a la temperatura ambiente.
En la balanza de vicios y virtudes del horno en zigzag, éstas pesan más que aquéllas, tanto bajo el punto de vista técnico como en el aspecto económico, y esta inclinación del flel ha sido la causante de su aceptación en la industria.

\section{CALCULOS TERMICOS EN EL PROYECTO}

Vamos a considerar el caso más general, es decir, un horno con extracción de aire caliente para aprovechamiento posterior. Para mayor sencillez supondremos el horno con un solo fuego. Si fuera de varios, el problema, con las ligeras variaciones procedentes de las pérdidas por transmisión, dependientes en cada horno de la distribución de temperaturas en él, se reduciría a multiplicar por $n$ los resultados obtenidos en el cálculo. Este cálculo no pretende ser riguroso, sino aproximado: con un grado de aproximación suficiente para proporcionar datos dignos de ser considerados como reales sin cometer errores groseros. Nos apoyaremos en algunos apartados en datos confirmados por la experiencia, que merecen crédito para este tipo de hornos y que simplifican notablemente las operaciones.

No se tiene en cuenta el período transitorio de puesta en funcionamiento o de encendido después de ser apagado por cualquier circunstancia. En este horno, que funciona continuamente, por su sencillez, solidez $y$ facilidad de manejo raramente se da una interrupción de marcha, por lo que estos períodos excepcionales se diluyen frente al tiempo de funcionamiento continuo.

Es cómodo referir los cálculos siguientes al intervalo de tiempo en el que, como término medio, se produce una cámara, ya que es el período de tiempo en el cual se repiten en el horno todos los fenómenos $\mathrm{y}$ distribución de temperaturas, aunque con una cámara de adelanto, naturalmente.

El intercambio de calor que en un hor- 
no tiene lugar en él y con el exterior, se reduce a los siguientes apartados:

a) Calor del combustible.

b) Calorías neçesarias para la evaporación de agua del material crudo.

c) Calorías necesarias para eliminación del agua de cristalización y zeolítica.

d) Calorías eliminadas por los humos, en forma de calor sensible y la tente.

e) Calorías eliminadas por paredes y suelo.

f) Calorías almacenadas en el material cocido al ser retirado del horno.

g) Calorías eliminadas con el aire de recuperación.

h) Calorias en el combustible no quemado.

Evaluados unos y otros conceptos, entre ellos deberá existir la igualdad entre las calorías introducidas en el horno $y$ las que salen de él:

$$
a=b+c+d+e+f+g+h .
$$

El rendimiento de la cocción, considerando como perdido el aire de recuperación, será:

$$
\rho_{1}=100 \frac{b+c}{a} .
$$

Si consideramos como aprovechamiento del horno las calorías del aire de recuperación, el rendimiento será:

$$
\rho_{2}=100 \frac{b+c+g}{a} .
$$

a) Calorias del combustíble introducido en el horno.

El carbón necesario para la cocción de $1 \mathrm{Tm}$ de cocidos es variable con la magnitud de los hornos, el elemento que se cuece y las condiciones particulares de la cocción. Sin embargo, esta variación oscila entre límites estrechos, siempre $y$ cuando el horno sea conducldo correctamente. En un estudio estadístico realizado por el Service en Usines de la S. P. P. F. T. C., en 120 hornos, dí́ unos resultados según los cuales el gasto oscilaba entre 40 y $75 \mathrm{~kg} / \mathrm{Tm}$ de cocidos. La

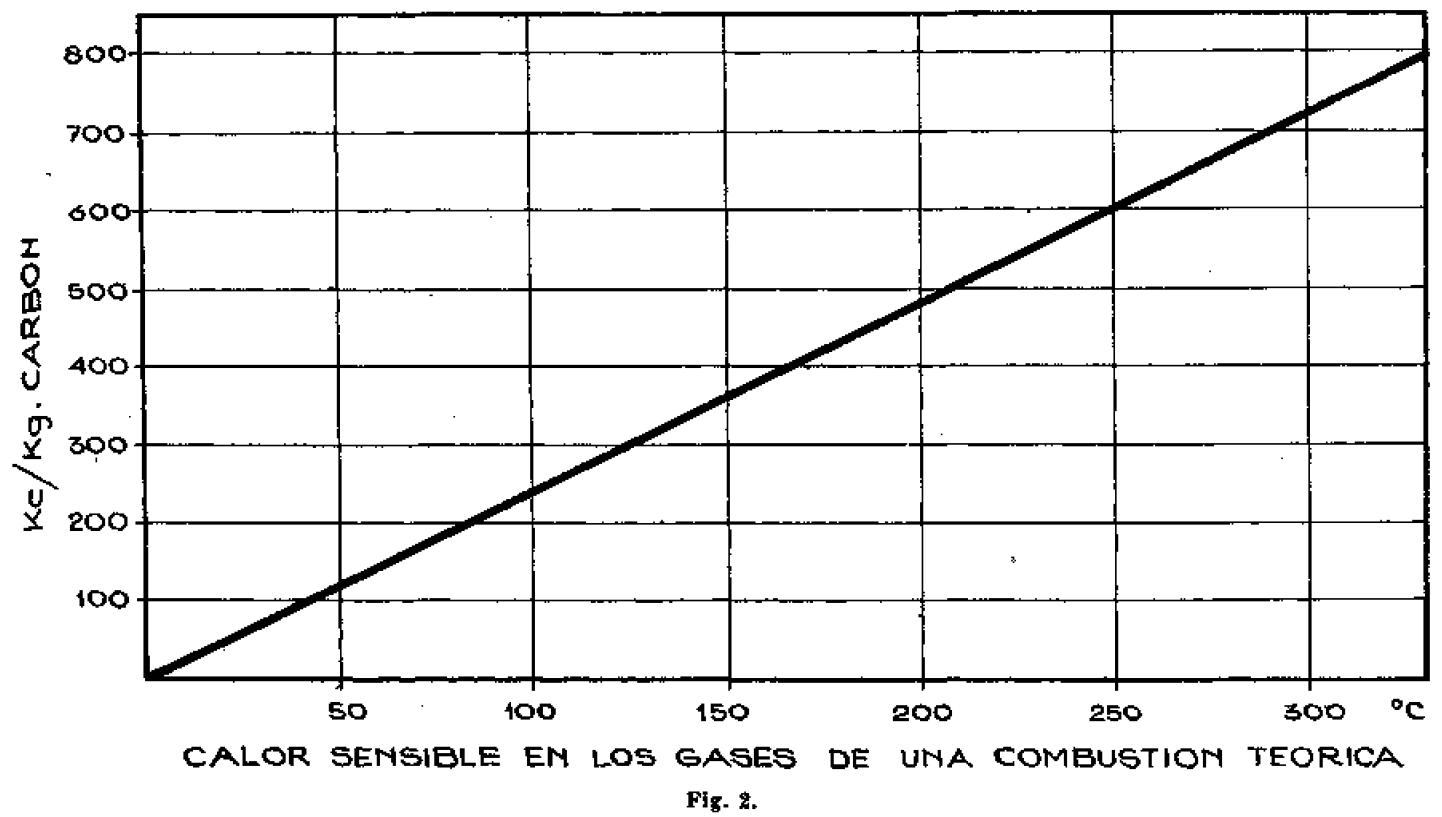


moda de la distribución de consumo correspondia a $48 \mathrm{~kg}$ de carbón $/ \mathrm{Tm}$ de cocidos, agrupándose la mayoría de los datos alrededor de éste, por lo que se pueden considerar los resultados alejados como anómalos. Los resultados están referidos a un carbón de 7.000 calorías de poder calorífico inferior. Normalmente se puede tomar esta cifra como consumo normal de un horno en primera aproximación, adaptando naturalmente el consumo en kilogramos de carbón, el que se vaya a emplear en cada caso, de forma que sea equivalente al número de calorías por unidad de productos cocidos.

El carbón empleado normalmente en estos hornos en España es un semigraso de poder calorífico cercano a los 6.000 $\mathrm{kg} / \mathrm{kcal}$ de carbón, valor que puede tomarse en los cálculos previos.

Conociendo concretamente el carbón que se va a utilizar, fácilmente puede hacerse el equivalente de calorías al consumo antes mencionado. El sustituir un carbón por otro, teniendo en cuenta únicamente que posean el mismo número de calorías totales por las de cocidos, puede hacerse, ya que las temperaturas exigidas en un horno para cerámica de construcción no son muy altas, y se alcanzan fácilmente, sobre todo cuando se dispone de un aire para la combustión en las excelentes condiciones en que está-precalentado-en los hornos zigzag cuando llega a las cámaras de combustión.

b) Calorías necesarias para la evaporación del agua del materlal procedente del secado.

Es un apartado que, para evaluar correctamente, habrá que tener en cuenta el cálculo del secadero y conocer las condiciones en que el material sale de él. El agua que puede tener al abandonar el secadero es muy variable, pudiendo oscilar, según los casos, entre un $2,5 \%$ y un $10 \%$, y todavía cifras más altas. Podemos considerar a falta de datos más concretos, una cifra del orden del $7 \%$ como más normal.

Las calorías perdidas por este concepto serán las necesarias para evaporar el agua y elevar la temperatura del vapor a la de los humos, que es la que abandona el horno.

Según Regnault, las calorías necesarias para elevar el agua de la temperatura $\theta$ -líquida-a la temperatura $t$-vapores :

$$
\mathrm{Q}=606,5+0,305 t-\theta \text {. }
$$

c) Calorias necesarias para la eliminación del agua de cristalización $y$ zeolítica de las areillas.

Como es sabido, las arcillas pierden, al ser calentadas, primeramente el agua de humedad a que nos hemos referido en el apartado anterior. Entre los $100 \mathrm{y} 250^{\circ} \mathrm{C}$ pierden el agua de cristalización; $\mathrm{y}$ entre los $350 \cdot$ y $700^{\circ}$, el agua zeolítica. Estos márgenes de temperatura son variables, dependiendo, para una misma arcilla, de la velocidad de calentamiento.

Los porcentajes (relativos entre sí y absolutos con respecto al producto) del agua de cristalización y zeolítica que tiene una arcilla dependen del tipo de arcilla. Estos valores en una arcilla de las utilizadas en cerámica pueden oscilar entre el $10 \mathrm{y}$ el $15 \%$. Como calor necesario para liberar este agua, puede tomarse como cifra media 10 a 15 kcal por $\mathrm{kg}$ de arcilla, cifra que, como se ve, es pequeña respecto al volumen de calorías necesarias para la cocción, por lo que no supone gran error tomar un valor medio.

\section{d) Calorías eliminadas por los humos.}

Los humos llevan almacenadas calorias en forma de energía potencial-calor latente $\longrightarrow$ en forma de energía cinética - calor sensible-.

El calor sensible puede determinarse conociendo el calor específico de los humos, el volumen de humos evacuado y la temperatura a que éstos salen al exterior.

El calor específico varía con la composición de los humos y con la temperatura. 
Es un factor difícil y engorroso de determinar.

La composición de los humos puede determinarse conociendo la composición elemental del carbón y el exceso de aire empleado en la combustión. Conocidos estos términos, y suponiendo una combustión perfecta, es sencillo hallar el calor específico de la mezcla a una temperatura, puesto que los calores específicos de los elementos de la combustión $\mathrm{CO}_{2} \cdot \mathrm{H}_{2} \mathrm{O}$, $\mathrm{O}_{2}$ y $\mathrm{N}_{2}$, despreciando los elementos que se encuentran en proporciones débiles -que podemos encontrar en multitud de tablas-, y calculando sus porcentajes en los humos, fácilmente podemos determinąr el calor específico medio de éstos. gráfica, que se puede utilizar sin dificultad alguna y que da unos resultados con un grado de aproximación aceptable. Se expone, a continuación, la curva hallada para un carbón de tipo medio, semigraso, de los utilizados normalmente en los hornos zigzag. Estas curvas pueden ser aplicadas a carbones de composición similar a la indicada, cometiéndose en estos casos errores mínimos.

Los calores específicos tomados en la confección del gráfico son los de $\mathbf{R}$. C. H. Hech Mechanical Engineering. Enero, 1950.

La temperatura a que se evacuan los humos no puede determinarse a priori,

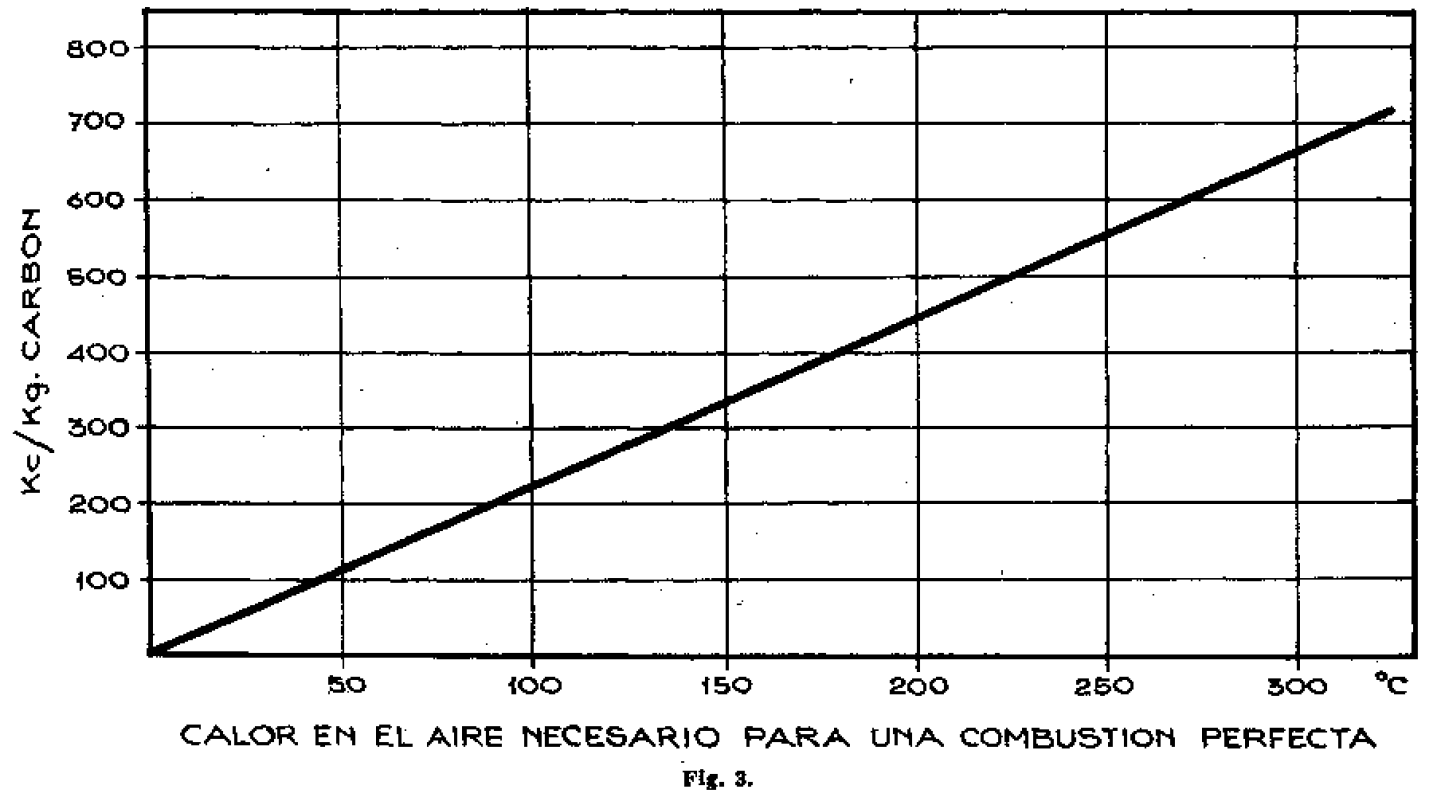

Sin embargo, esto, sencillo en teoría, es difficil de realizarse en la práctica, pues el análisis del carbón que vamos a utilizar-si lo conocemos -en la mayoría de los casos no nos dará resultados estimables, teniendo en cuenta la gran probabilidad que existe de que el carbón empleado a lo largo del tiempo varíe de composición entre amplios límites, de acuerdo con las disposibilidades que de él existan.

Afortunadamente, con frecuencia es posible traducir estos cálculos en forma salvo con groseras aproximaciones. Haciendo el estudio del horno como regenerador, tal como se indica más abajo, obtendremos una cifra, que se aproxima bastante a la realidad. En cada caso dependerá no solamente del horno, sino de la forma y estado — en especial humedadde los productos introducidos en el horno.

En el gráfico anterior no está incluído el vapor de agua que, procedente del carbón con que se alimenta el horno, acompaña a los humos y que supone una cifra 
aceptable. En este horno de marcha continua puede determinarse aquél fácilmente y referirse a la unidad de volumen de humos expulsados al exterior, o al kilo de combustible, según interese. Suponiendo que todo él se introduce en forma líquida a la temperatura ambiente-hipótesis que será la exacta en la mayoría de los casos-, las calorías que este vapor ha absorbido pueden calcularse fácilmente por la fórmula de Regnault.

A estas calorías, calculada para el caso de una combustión térica, hay que añadir las calorías evacuadas por el aire que procedente del exceso de aire para la combustión se elimina con los humos, y que no habiendo tenido más misión que faci\itar la reacción completa del combustible con el oxígeno, se ha calentado y ha almacenado calorías lo mismo que los humos. Se da, a continuación, una curva con las calorías almacenadas por el aire en función de la temperatura a que se halla y tomando como unidad el aire teóricamente necesario para la combustión. Sumando estas calorias, multiplicadas por el porcentaje correspondiente al exceso de aire de combustión a la curva de la figura (2), obtendremos las calorías que en forma de calor sensible arrastran los gases secos.

El calor latente de los gases, es, en general, difícil de predecirse. Este calor es el que llevan los humos en forma de inquemados, principalmente $\mathrm{CO}, \mathrm{H}_{2}$ y $\mathrm{CHn}$. Puede suponerse, con bastante aproximación según los datos de la S. P. P. F. T. C., antes mencionados, que las pérdidas por Ios humos son del orden de $10 \mathrm{~kg}$ de carbón de $7.000 \mathrm{kcal}$, por tonelada de productos cocidos.

\section{e) Pérdidas por las paredes y el suelo.}

Parte de las pérdidas por las paredes, al exterior, se efectúan por radiación, desde la parte externa del muro al ambiente.

Como es sabido, todo cuerpo emite radiaciones caloríficas, cuya intensidad es proporcional a la cuarta potencia de su temperatura absoluta y a la superficie del cuerpo, dependiente la constante de proporcionalidad de las características particulares a la superficie del cuerpo que irradia.

A la vez, un cuerpo que irradia es irradiado por los que están contiguos a él. Cuando el calor perdido por radiación es superior al recibido, el cuerpo se enfría; se calienta cuando el calor recibido es mayor, y permanece a temperatura constante cuando hay igualdad entre las radiaciones emitidas $\mathrm{y}$ absorbidas. De acuerdo con ello, el calor emitido por radiación por una pared al ambiente será proporcional a la diferencia de las cuartas potencias de las temperaturas absolutas que existen en la pared y en el ambiente.

Aparte de la perdida anterior, un cuerpo en contacto con un flúido pierde calor por convección. Las pérdidas por convección son difíciles de determinar, pues dependen de múltiples factores; $y$ en el caso concreto de un horno al aire libre, oscilará con la velocidad del aire que-como puede comprenderse en extremo-es variable $y$ de difícil previsión.

Juntamente con las pérdidas por conducción entre la pared y el aire, éstas son las formas en que las paredes ceden calor al ambiente, calor cuya determinación es compleja si se quiere hacer con exactitud.

Si consideramos la pared de un horno, que tiene la cara interior a una temperatura $T_{1}$ y la exterior a una temperatura $\mathrm{T}_{o}$, las pérdidas a través de ella vienen dadas por la expresión:

$$
\mathbf{Q}=\mathbf{C} \cdot h \cdot \mathrm{s} \frac{\mathbf{T}_{1}-\mathbf{T}_{a}}{e}
$$

siendo:

$$
\begin{aligned}
& \mathrm{C}=\text { coeficiente de transmisión. } \\
& h=\text { tiempo. } \\
& s=\text { superficie. } \\
& e=\text { espesor de la pared. }
\end{aligned}
$$

La temperatura $T_{i}$ no es igual a la del horno, sino ligeramente inferior. Tampo- 
co la temperatura $\mathbf{T}_{o}$ es igual a la del ambiente, sino que differe en general de ella, dependiendo esta diferencia de la facilidad con que esta cara cede su calor al ambiente por conducción, convección $\mathrm{y}$ radiación.

Heiligenstaedt propone la fórmula:

$$
\mathrm{Q}=\frac{\mathrm{T}_{i}-\mathrm{T}_{\mathrm{a}}}{\frac{1}{\mathrm{~K}}+\frac{\mathrm{S}}{\mathrm{C}}}
$$

en la que:

$T_{a}$ es la temperatura ambiente.

$\mathbf{K}$ es el coeficiente que puede tomarse como $\mathrm{K}=7,286+0,01632 \mathrm{~T} \mathrm{kcal} /$ $\mathrm{m}^{2} \mathrm{~h} \cdot \mathrm{C}$.

$\mathrm{T}_{i}=$ Temperatura de la cara caliente de la pared.

$\mathbf{S}=$ espesor del muro.

$\mathrm{C}=$ conductividad térmica de la pared.

En general, un muro de un horno no estará compuesto de un solo material, sino que en el intermedio de las paredes interiores y exteriores existirá un relleno de un material aislante que-como se dice más adelante--suele ser arena.

Puede sustituirse el muro, a los efectos del cálculo de las pérdidas calóricas, por un muro equivalente, de forma que, estando formado por un solo material, tenga un espesor tal que las pérdidas sean equivalentes a las reales.

En nuestro caso, considerando que es arena el material empleado como aislante, podemos sustituir el espesor de la arena -arena seca-por otro de ladrillo que sea aproximadamente dos veces y media mayor.

La conductividad térmica depende también de la temperatura. Para un horno de cocción de cerámica de construcción, las temperaturas alcanzadas en él-y que en principio podemos suponer iguales a las de la pared interior-oscilan entre una temperatura muy próxima a la ambiente y $\operatorname{los} 950^{\circ} \mathrm{C}$-cifra media que habría que corregir en cada caso, según la temperatura de cocción-. Admitiendo que la distribución de temperaturas en el interior de la pared es lineal, que la temperatura de la cara exterior es la ambiente-lo que, en nuestro caso, se acerca mucho a la realidad-y teniendo en cuenta que la conductividad varía sensiblemente en correspondencia lineal con la temperatura, podemos tomar como conductividad media la conductividad del material a $500^{\circ} \mathrm{C}$ -aproximadamente la temperatura media entre las dos caras de la pared-. El valor de la conductividad de los ladrillos a esa temperatura es de $1,07 \mathrm{kcal} \cdot \mathrm{m} / \mathrm{m}^{2}$. . $\mathrm{h}^{\circ} \mathrm{C}$.

Considerando cada cámara por separado, ésta efectúa un calentamiento y enfriamiento periódico. La inercia térmica de las. paredes impide que la distribución de la temperatura en ella se efectúe de forma lineal, $\mathrm{y}$, por tanto, las pérdidas calóricas no pueden calcularse con la fórmula anterior, sino que se aleja bastante de la realidad.

Según los estudios de la Transactions American Society of Mechanical Engineering, para hornos en trabajo intermitente aplicados a este caso, el calor perdido por una cámara en un ciclo completo es aproximadamente el $50 \%$ del que perdería si estuviese trabajando continuamente-calculable por las fómulas anteriores-. De este calor, aproximadamente el $40 \%$ puede suponerse que se recupera con el aire de recuperación.

Las pérdidas por el techo del horno pueden calcularse, sin gran error, de forma análoga a las pérdidas por las paredes, y lo mismo puede hacerse con el suelo del horno en el caso de ser un suelo ventilado.

En el caso de solera no ventilada, es decir, en contacto directo con el suelo, o fundaciones sin intermedio de una cámara de aire, la evaluación de las pérdidas requiere un cálculo aparte. Este cálculo, en general, es complejo si se quiere efectuar con todo rigor, más todavía en hornos intermitentes. Las pérdidas no son regulares en toda la extensión de la solera, sino que varían ostensiblemente según la situación del punto considerado. 
Como se comprende intuitivamente, las pérdidas en los puntos próximos a los ángulos son superiores a las perdidas en el centro de la cámara.

La pérdida de calor por el suelo es proporcional-para cámaras de la misma forma geométrica-a las dimensiones lineales de la solera y no a la superficie, como ocurre en las paredes. Trinks indica la fórmula

$$
Q=-\frac{\mathbf{S} \cdot \mathbf{C} \cdot \mathbf{A}\left(\mathbf{T}_{i}-\mathbf{T}_{\mathrm{q}}\right)}{\mathbf{D}}
$$

para las pérdidas por el suelo en un horno continuo. En ésta

$\mathbf{S}=$ factor de forma que para cada cámara del horno puede tomarse $\mathrm{S}=\mathbf{0 , 3 3}$.

$\mathrm{C}=$ conductividad de $\operatorname{los}$ materiales en $\mathrm{kcal} / \mathrm{h} \cdot \mathrm{dm}^{2} \cdot \mathrm{h}^{\circ} \mathrm{C}$.

$\mathrm{A}=$ superficie del suelo en $\mathrm{dm}^{2}$.

$\mathrm{T}_{i}=$ temperatura del horno (en la parte inferior).

$\mathrm{T}_{a}=$ temperatura ambiente.

$\mathrm{D}$ = la menor dimensión de la solera en $\mathrm{dm}$.

Para un horno en zigzag, en el que la cámara trabaja intermitentemente, se puede hacer una reducción análoga a las pérdidas por las paredes.

Como primera aproximación se puede tomar como media aproximada de las pérdidas el $75 \%$ de las pérdidas que tendría una pared la misma superficie interior que la solera y de un espesor igual a la sexta parte del ancho de ésta.
La expresión adoptada para la pérdida por las paredes, se reflere al caso en que

estas paredes tengan dimensiones infinitas. No obstante, como pequen̄o error puede aplicarse a las paredes normales, introduciendo posteriormente la corrección de las pérdidas de calor por las esquinas.

Langmuir propone la siguiente fórmula para calcular las pérdidas en régimen permanente en un muro:

$$
Q=C\left(T_{i}-T_{e}\right)\left[\frac{A}{S}+0,54 S+n 1,25 s\right] ;
$$

fórmula hallada teóricamente y corregida con experiencias posteriores, en la que:

$$
\begin{aligned}
& \mathrm{C} \text { - conductividad media del muro } \\
& \left(\mathrm{kcal} / \mathrm{h} \cdot \mathrm{m} \cdot{ }^{\circ} \mathrm{C}\right) \text {. } \\
& \mathbf{T}_{i}^{\prime} \text { = temperatura de la pared interior }
\end{aligned}
$$

(Continuará.) 